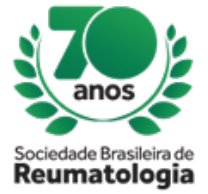

\title{
A CASE OF TOLOSA-HUNT SYNDROME IN A PATIENT WITH SYSTEMIC LUPUS ERYTHEMATOSUS
}

Micheline Sulzbacher Batista (Hospital de Clinicas de Porto Alegre, Porto Alegre, RS, Brasil), Afonso Papke (Hospital de Clinicas de Porto Alegre, Porto Alegre, RS, Brasil), Bruna Lima Porto (Hospital de Clinicas de Porto Alegre, Porto Alegre, RS, Brasil), Victoria Silveira Carvalho (Hospital de Clinicas de Porto Alegre, Porto Alegre, RS, Brasil), Ana Laura Fischer Kunzler (Hospital de Clinicas de Porto Alegre, Porto Alegre, RS, Brasil), Maurício Simoni Candaten (Hospital de Clinicas de Porto Alegre, Porto Alegre, RS, Brasil), Nicole Pamplona Bueno de Andrade (Hospital de Clinicas de Porto Alegre, Porto Alegre, RS, Brasil), Claiton Viegas Brenol (Hospital de Clinicas de Porto Alegre, Porto Alegre, RS, Brasil)

\section{BACKGROUND}

The Tolosa-Hunt Syndrome (THS) refers to painful ophthalmoplegia associated with paresis of one or more of the cranial nerves (III, IV, V and VI) due to non-specific granulomatous inflammation in the cavernous sinus. It is a rare condition whose incidence has been estimated approximately one case per million per year, may present at any age and without sex predilection. Typically, it is unilateral and responds dramatically to steroids. The characteristic MRI findings are enlargement of the cavernous sinus by abnormal tissue with contrast enhancement maker. Although cases of THS have been reported in patients with other inflammatory disorders, this is a rare ophthalmic complication of systemic lupus erythematosus (SLE).

\section{CASE REPORT}

A 48-year-old female with a previous diagnosis 20 years ago of SLE (SLE-SLICC classification criteria: positive ANA, positive anti-SM antibody, polyarthritis, photosensitivity) was adequately controlled with disease modifying anti-rheumatic drugs. In mid of 2018, she presented intense right periorbital pain and ipsilateral frontal headache, palpebral ptosis, diplopia, divergent strabismus and hypoesthesia of the right side of the face with 7 days of evolution. Fever peak isolated 15 days ago. Physical examination showed involvement of the ophthalmic and maxillary branches of the trigeminal nerve, VI and III cranial right pairs, indicating topographically involvement of the cavernous sinus. Ophthalmologic examinations revealed no pathologic findings. MRI reveals a tenuous thickening with isosignal at T1 in topography of the right cavernous sinus, in addition to signs of intracranial hypertension and microangiopathy. A lumbar puncture evidenced increase in CSF pressure and analysis of cerebrospinal fluid excluded infectious causes. A diagnosis of presumed THS in a patient with SLE was made and treated with $1 \mathrm{mg} / \mathrm{kg} /$ day of methylprednisolone with dramatic improvement of palpebral ptosis and headache in less than 48 hours, maintaining a discreet strabismus. In 8 weeks complete resolution of the clinical condition. Furthermore, the patient had activity of SLE, with anti-DNA positive, hypocomplementemia, elevated acute phase reactants, anemia and mild proteinuria. Due to concomitant SLE activity, azathioprine was started in addition to a gradual reduction of corticosteroids. The patient did not present relapse in follow-up.

\section{CONCLUSION}

In summary, still remains undefined if THS can be considered a manifestation of systemic inflammatory disease or may simply represent an association of the two autoimmune conditions. 